\title{
The Charcot Foot: An Emerging Public Health Problem for African Diabetes Patients
}

\author{
Zulfiqarali G Abbas ${ }^{1,2}$, JK Lutale ${ }^{1}$, C Formosa ${ }^{3,4}$, A Gatt ${ }^{3,4}$, \\ N Chockalingam ${ }^{4,3}$
}

\footnotetext{
${ }^{1}$ Department of Internal Medicine, Muhimbili University of Health and Allied Sciences, Dar es Salaam, Tanzania

${ }^{2}$ Department of Internal Medicine, Abbas Medical Centre, Dar es Salaam, Tanzania

${ }^{3}$ Faculty of Health Sciences, University of Malta

${ }^{4}$ Centre for Biomechanics and Rehabilitation Technologies, Staffordshire University.
}

Corresponding Author:

Professor Nachiappan Chockalingam

email: N.Chockalingam@staffs.ac.uk

Address:

Centre for Biomechanics and Rehabilitation Technologies,

Staffordshire University,

Leek Road,

Stoke on Trent, ST4 2DF

United Kingdom. 


\begin{abstract}
Background: Although the awareness, diagnosis, management of the complications associated with diabetes have improved in African countries over the past decade, surveillance activities in Tanzania and anecdotal reports from other African countries have suggested an increased prevalence of Charcot Neuroarthropathy $(\mathrm{CN})$ over the past few years.
\end{abstract}

Aim: To characterize the epidemiology and the clinical burden of $\mathrm{CN}$ in a large diabetes population in Tanzania, and to evaluate outcomes of persons with the condition.

Methods: This was a prospective analytic cohort study conducted between January 2013 through December 2015. Following informed consent, patients were followed at the outpatient clinic. Detailed clinical assessments and documented presence of diabetic peripheral neuropathy (DPN), macrovascular disease and microvascular disease were recorded. Education and counselling were part of the follow-up program.

Results: 3,271 ulcerations were presented at the clinic during the 3-year study period. 571 (18\%) met the case definition for $\mathrm{CN}$; all patients had Type 2 diabetes. The prevalence for each of the years 2013, 2014, and 2015 was 19/1192 (1.6\%), 209/1044 (20\%), and 343/1035 (34\%), respectively; the increases in the slope of the trendline was statistically significant $(\mathrm{p}<0.001)$

Conclusion: The prevalence of $\mathrm{CN}$ is increasing in the Tanzanian diabetes patient population, and is strongly associated with neuropathy. CN can lead to severe deformity, disability, and amputation. Due to the risk of limb amputation, patients with diabetes must seek immediate care if signs or symptoms appear and avoid delay in seeking medical attention. Early diagnosis of $\mathrm{CN}$ by caregivers is extremely important for successful outcomes.

Keywords: Charcot Neuroarthropathy, Africa, Diabetic foot ulcer, diabetic peripheral neuropathy, peripheral arterial diseases, infection, amputation, mortality 


\section{Introduction:}

The United Republic of Tanzania is a country in Sub-Saharan Africa (SSA), with a population of 60 million people. SSA is experiencing a rapid increase in the prevalence of diabetes. In 2019, a report from the international diabetes federation estimated that there were 19 million people with diabetes in SSA and should the current trend continue, the overall prevalence is projected to be 47 million by 2045 , which is an increase of $143 \%^{1,2}$.

Diabetic Peripheral Neuropathy (DPN) is one of the most common complications affecting patients with diabetes in both developed and less developed countries. The latest pooled prevalence rate of DPN across Africa is reported to be $46 \%$ with rates ranging between $7.5 \%$ and $83.4 \%{ }^{3}$. Previously, Abbas et al found no differences in the rates of DPN between African and Asian patients with DFU ${ }^{4}$. Also, another old comparative study found no significant differences in the presence of DPN in $80 \%$ of inpatients with DFU in Tanzania, Germany and India ${ }^{5}$. Although awareness, diagnosis, management of the complications associated with diabetes have improved in African countries over the past decade, surveillance activities in Tanzania and anecdotal reports from other African countries have suggested an increase in the prevalence of Charcot Neuroarthropathy $(\mathrm{CN})$ over the past few years. DPN leads to more severe complications including $\mathrm{CN}$, a limb threatening condtion that could become a life-threatening condition in a low resourced setting leading to the destruction of bones and joints of the foot ${ }^{2}$. A foot deformity, therefore, is often accompanied by ulceration, infection and gangrene thus increasing the risk of amputation ${ }^{2}$. This is of concern because of the potential for $\mathrm{CN}$ to set back the positive progress, and outcomes, including reducing the rates of leg amputations, achieved through the Step-by-Step foot program in Tanzania ${ }^{6}$.

Therefore, it becomes important to characterize the epidemiology and clinical burden of $\mathrm{CN}$ in a large diabetes population in Tanzania and to evaluate the outcomes of persons with this condition. 


\section{Methods:}

This was a prospective cohort study. Data was collected of 3,271 ulcerations in 1586 consecutive patients with Type 2 diabetes who attended the clinic with diabetic foot ulcers over a period of three years from January 2013 to December 2015. Ethical approval to conduct this study was sought and granted by the local ethics committee. Core details of all people with diabetes attending a single specialist service in Dar es Salaam, for the management of diabetic foot ulcers (DFUs) in Tanzania have been reviewed. Patients with DFU were prospectively recruited. The centre serves a population of approximately 7 million people of mixed ethnicity (African, Indian Asian and Arab). Only the first-ever episode of foot ulceration caused in conjunction with $\mathrm{CN}$ during the study period was recorded as an individual case of $\mathrm{CN}$ with an ulcer; multiple ulcers occurring at the same time were counted as a single episode of ulcer.

From this database, patients were selected for inclusion in this study if they met the clinical definition of $\mathrm{CN}$ and were diagnosed with this condition for the first time by their physician. Given the limited resources available in these remote areas, patients were selected if they exhibited a high degree of clinical suspicion of $\mathrm{CN}$. The clinical definition of $\mathrm{CN}$, includes DPN, joint swelling and dislocation, reported pathological fractures, severe deformity (loss of medial longitudinal arch), signs of inflammation, swelling of the foot, increased local temperature of the skin and redness ${ }^{7}$.

Following informed consent, patients were followed up at the outpatient's clinic, with data being collected until the end of the study period or the death of the patient. Data collected included demographics, such as age, BMI, duration of diabetes, presence of foot ulcers, ulcer stage (Wagner grade), reason of ulceration, healed/unhealed ulcers. In addition, temperature was measured using infrared thermometry. Due to resource constraints, a foot $\mathrm{x}$ ray was not done in all patients. 


\section{Clinical assessment:}

We carried out a detailed clinical assessment and documented the presence of loss of protection sense or DPN and peripheral arterial diseases (PAD) using estabished clinical protocols.

$D P N-:$ During the clinical examination, patients were assessed for DPN using the $10 \mathrm{~g}$ Semmes-Weinstein monofilament on the plantar aspect of the foot, utilizing the 5-point test method, including the $1^{\text {st }}, 3^{\text {rd }}, 5^{\text {th }}$ metatarsal heads, hallux and heel ${ }^{8}$. Any patient who was unable to feel the monofilament on one or more sites was classified as neuropathic ${ }^{8}$. Tests using a biothesiometer and hot and cold test (Sensisometer) were also conducted on every patient who attended the clinic during the study period.

$P A D-:$ As per standard clinical protocol pulses palpated included Dorsalis pedalis and Posterior tibalis ${ }^{8}$. These were graded as normal if both pulses were felt as good bounding pulses. If only one pulse was felt or felt with decreased volume, or if no pulse was felt during the examination this was noted as some form of arterial impairment to the foot. Doppler tests were only done if pulses were not felt. Some patients who could afford CT Scan angiography were analysed using this method.

Ulcer classification-: In the presence of ulcers, each ulcer was documented separately, detailing the cause of ulceration, classification of ulcerations using the Wagner grading 9,10 whether ulcers had undergone sloughectomy and those which resulted in major or minor amputations.

Education and counselling-: Education and counselling were part of the follow-up program. Every high risk patient (DPN, PAD and history of past ulcer or amputation) was given 
education either on a one to one basis or in a group ${ }^{6}$. The education was weekly and importance was given to footwear and offloading. Education included the following recommendations: Look at feet every day, learn to look for deformity and complications, learn to take care of the diabetic skin, learn to take care of nails, what to look for in socks, what to look in footwear, and identify problems and act fast ${ }^{6}$. Become familiar with changes associated with bacterial or fungal infection. Educational material was also distributed amongst patients.

\section{Results:}

Out of 3,271 ulcerations presenting at the clinic, 571 (18\%) ulcerations in 226 (14.24\%) Type 2 diabetes patients with a positive diagnosis of Charcot neuroarthropathy, were identified during the 3year study period. 2700 ulcers were attributed to 1360 noncharcot patients. Out of the 1586 patients reported within the study, in 2013, there were 17 CN patients and 517 patients without CN. This increased to 86 and 374 patients in 2014 and 123 and 469 in 2015.

Patient demographics: median age 56 years (range between 14 to 92 years), the median duration of diabetes was 10 years (range 1-33 years); median BMI $26.6 \mathrm{~kg} / \mathrm{m}^{2}$ (range $18-49 \mathrm{~kg} / \mathrm{m}^{2}$ ). With regards to microvascular disease, none had retinopathy or nephropathy. Delay in seeking medical treatment was common (median 7 days (range 1-360 days). Five patients were on diet only, 144 were on oral hypoglycaemic agents, 76 were on insulin and 1 patient was on both oral medication and insulin.

All presenting cases had active ulcerations, all of whom had a history of previous ulcers, with some of them presenting with multiple ulcerations. This amounted to 571 new ulcerations over the 3 years. During this period, 19 ulcers were diagnosed in 2013, 209 ulcerations in 2014 and 343 ulcerations in 2015 (Table 1, Figure 1), indicating a statistically significant increase in the slope of the trend line $(\mathrm{p}=0.001)$. 
<table 1 here >

<figure 1 here $>$

Figure 1: Prevalence of $\mathrm{CN}$ ulcers during the study period

One hundred and forty-seven ulcerations were neuro-ischaemic and 424 were purely neuropathic in nature. At presentation, 397 ulcers (70\%) were classified as Wagner grade 2, $72(12 \%)$ as Wagner grade 3 and $102(18 \%)$ were Wagner grade 4. Table 2 reports ulcer demographics, including those that healed and those that did not heal until the end of the study period. Over $90 \%$ of these ulcers were presented in the midfoot region. Common causes of ulceration included blisters (36\%), callus (13\%), prick with a sharp object $(9.3 \%)$, rat bites $(9 \%)$, blunt trauma $(7 \%)$ and burns $(4 \%)$. The majority of ulceration underwent sloughectomy $(90 \%)$ as part of the medical treatment. Delay in seeking medical treatment was common amongst this cohort, with a median of 7 days, ranging between 1 to 360 days before initial assessment.

<table 2 here >

In this cohort of 571 ulcers, $395(69.2 \%)$ healed during the study period, taking a mean of 141 days (SD 62days). The mean age of patients with healed ulcers was $55.7 \%$ (SD 12.01) with a mean fasting blood glucose of 13.61 (SD 8.4) $\mathrm{mmol} / \mathrm{L}$ and 11.1 (SD7.2) years mean duration of diabetes. One hundred seventy-six (30.8\%) ulcers remained unhealed by the end of the study period. The mean age of these patients was 53.4 (SD13.83) years, who had a mean fasting blood glucose of 13.89 (SD8.24) mmol/L, which is quite comparable to those of the healed cohort. Duration of diabetes was 10.66 (SD8.05) years, which is also 
comparable to that of the healed cohort. Out of these unhealed ulcers, 54 patients $(23.9 \%)$ from the 226 patients who presented at the clinic with $\mathrm{CN}$ sustained some form of amputation. There were no amputations in 2013, $36(66.7 \%)$ in 2014 and $18(33.3 \%)$ in 2015.

\section{Discussion:}

The results from this study demonstrate the increasing prevalence of $\mathrm{CN}$ ulcerations in Tanzania. Although in 2015 fewer cases of diabetic non - CN ulcerations were seen at the diabetic clinic, there was a significant increase in the number of $\mathrm{CN}$ presenting with ulcerations at the first visit. We observed an exponential increase (from 3.2 to 21 percent) of $\mathrm{CN}$ patients between the first and the second year within the study period. Although this is not attributed to any specific reason, the trend in increased $\mathrm{CN}$ was consistent over the 3 years of the study period. The median duration of diabetes amongst this population was of 10 years, confirming that it is commonest in those patients with long-term diabetes. Whilst it might be impossible to totally prevent diabetic foot ulceration, it is certainly feasible to prevent the progression of small ulceration progressing into more complicated wounds, and possibly $\mathrm{CN}$ in the presence of peripheral neuropathy. This study has shown that the prevalence of $\mathrm{CN}$ increased over the 3 years and those patients who presented with lower ulcer classification managed to heal, whilst those that presented with higher ulcer grading had more difficulty in healing. Due to this fact diabetes patients in Tanzania should be alerted on the importance of seeking medical help during the early stages of foot-related ulcerations and, healthcare professionals should instigate a proper investigation as to the aetiology of the increasing incidence of $\mathrm{CN}$ in Africa.

Recognizing this condition at an early stage encourages timely initiation of appropriate therapy, thus reducing the burden of this chronic condition both on the patient and health care systems. However, various challenges prevent this early detection in Tanzania 
especially amongst the poorer populations who cannot seek private medical attention. Foremost amongst these challenges are the huge, sometimes insurmountable, distances that patients would have to travel - mostly on already-afflicted feet - in order to cover the great distances from rural areas, where medical treatment may not be available. Poverty, unhygienic conditions, the purchase of appropriate footwear and the common practice of walking barefoot in rural communities ${ }^{2,11}$, are also often inevitable challenges that these patients face daily.

In this study, the median age was of 56 years, which is in concordance with other reported literature which highlight that CA presents in the $5^{\text {th }}$ and $6^{\text {th }}$ decade of life ${ }^{12,13}$. This is also similar to another study performed in a neighbouring SSA country ${ }^{12}$. Although in this study $\mathrm{CN}$ was reported to be equally distributed between midfoot and forefoot ${ }^{12}$, in our population $90 \%$ of all ulcers presented in the midfoot. The ulcers that healed presented on a lower Wagner grading when compared to those that did not heal. Indeed, there were $74.2 \%$ Grade 2 vs $59.1 \%$ between the healed and non-healed groups respectively, and $15.2 \%$ vs 23.9 Wagner Grade 4, respectively. These statistics indicate that the lower the Wagner grading of the ulcer, the more probability of healing, advocating for the need for early intervention at the initial stages of the ulcer. However, as discussed earlier, there may be significant challenges that contribute to delay in seeking medical attention.

To augment these barriers to timely diagnosis and treatment, acute $\mathrm{CN}$ may often be misdiagnosed for cellulitis, sprain, gout or deep venous thrombosis (DVT) due to lack of available facilities in centres in Tanzania. This emphasises the importance of additional training for health care professionals to help them to recognize the early signs and symptoms of this condition. Health care professionals, where there is only basic diagnostic equipment ${ }^{12}$, should be encouraged to routinely screen feet of diabetic patients and to refer promptly to 
more specialist diabetic services upon suspicion of $\mathrm{CN}$. Also, within this paper, we report that none of the patients had retinopathy or nephropathy. One could perceive this observation as unusual. However, it needs to be highlighted that these tests were not completed as a part of this study and they were independently carried out as a part of the wider healthcare system in Tanzania. The system requires the patients to complete these tests in different places. Nephropathy was tested by the levels of Serum Creatinine and Serum urea within a recognised hospital facility. The Eye department within the public hospital checked and reported for retinopathy using an ophthalmoscope. Whilst for this paper, we report the results as presented to us, future studies should make a provision for structured and thorough investigations into these topics. In addition, lack of disease confirmation using foot $\mathrm{x}$-rays in many paitents within study could be seen as a limitation.

The study was based on primary data gathered prospectively, hence allowing the timely collection of the parameters of interest. This study was conducted in one centre; the scenario in more rural areas where no such centres exist was not investigated and could draw a more morbid picture with regards to the prevalence of $\mathrm{CN}$ since in African countries, medical insurance or reimbursement of medical expenses are not always available. Amongst the poorer populations, such medical care could cost more than 2 years of their average income ${ }^{14}$, hence making it very difficult for patients to seek immediate help. Access to more advanced imaging modalities was limited, both logistically and financially, however several cases presented to the clinic in the acute phase where it is well known that radiological changes in this condition may be relatively difficult to appraise.

\section{Conclusion:}

$\mathrm{CN}$ amongst diabetes patients in Tanzanian is increasing in prevalence and develops as a result of neuropathy. $\mathrm{CN}$ can lead to severe deformity, disability, and amputation. Due 
to its seriousness, it is important that patients with diabetes seek immediate care if signs or symptoms appear and avoid delay in seeking medical attention. Early diagnosis of $\mathrm{CN}$ by caregivers is extremely important for successful outcomes. Warmth to the touch, redness and localized swelling with tenderness in the feet are key clinical findings that might herald a $\mathrm{CN}$.

\section{Acknowledgements:}

\section{Conflict of interest}

The authors report no conflict of interest.

\section{References}

1. International Diabetes Federation, Diabetes Atlas, 9th edition, 2019. Available from: https://www.diabetesatlas.org/en/resources/ Accessed 24 ${ }^{\text {th }}$ June 2021.

2. Abbas ZG. The diabetic foot worldwide: Sub-Saharan Africa. In: Boulton AJM, Rayman G, Wukich D (eds): The Foot in Diabetes 5th edn. John Wiley \& Sons Ltd, Chichester, UK; 2020:379-85.

3. Shiferaw, W.S., Akalu, T.Y., Work, Y. et al. Prevalence of diabetic peripheral neuropathy in Africa: a systematic review and meta-analysis. BMC Endocr Disord 2020;20:49. https://doi.org/10.1186/s12902-020-0534-5

4. Abbas ZG, Lutale JK, Archibald LK. Diabetic foot ulcers and ethnicity in Tanzania: a contrast between African and Asian populations. Int Wound J. 2009 Apr;6(2):124-31.

5. Morbach S, Lutale JK, Viswanathan V, Möllenberg J, Ochs HR, Rajashekar S, Ramachandran A, Abbas ZG. Regional differences in risk factors and clinical presentation of diabetic foot lesions. Diabet Med. 2004;21(1):91-5.

6. Abbas ZG, Lutale JK, Bakker K, Baker N, Archibald LK. The 'Step by Step' Diabetic Foot Project in Tanzania: a model for improving patient outcomes in less-developed countries. Int Wound J. 2011 Apr;8(2):169-75.

7. Rogers LC, Frykberg RG, Armstrong DG, Boulton AJ, Edmonds M, Van GH, Hartemann A, Game F, Jeffcoate W, Jirkovska A, Jude E, Morbach S, Morrison WB, Pinzur M, Pitocco D, Sanders L, Wukich DK, Uccioli L. The Charcot foot in diabetes. Diabetes Care. 2011 Sep;34(9):2123-9.

8. International Working group on the Diabetic Foot (IWGDF). The development of global consensus guidelines on the management and prevention of the Diabetic Foot 2019, www.iwgdf.org. https://iwgdfguidelines.org/guidelines/guidelines/ Accessed $24^{\text {th }}$ June 2020 
9. Abbas ZG, Lutale JK, Game FL, Jeffcoate WJ. Comparison of four systems of classification of diabetic foot ulcers in Tanzania. Diabet Med. 2008 Feb;25(2):134-7.

10. Camilleri A, Gatt A, Formosa C. Inter-rater reliability of four validated diabetic foot ulcer classification systems. J Tissue Viability. 2020 Nov;29(4):284-290. doi: 10.1016/j.jtv.2020.09.002.

11. Abbas ZG, Lutale JK, Archibald LK, Jeffcoate WJ. Rat bite as a cause of diabetic foot ulcer in sub-Saharan Africa. Int Wound J. 2020 Aug;17(4):897-899.

12. Wanzou JPV, Sekimpi P, Komagum JO, Nakwagala F, Mwaka ES. Charcot arthropathy of the diabetic foot in a sub-Saharan tertiary hospital: a cross-sectional study. J Foot Ankle Res. 2019 Jun 14;12:33. doi: 10.1186/s13047-019-0343-0.

13. Fauzi AA, Chung TY, Latif LA. Risk factors of diabetic foot charcot arthropathy: a case control study at a Malaysian tertiary care centre. Singapore Med J. 2016. 57(4):198-203

14. Cavanagh P, Attinger C, Abbas Z, Bal A, Rojas N, Xu ZR. Cost of treating diabetic foot ulcers in five different countries. Diabetes Metab Res Rev. 2012 Feb;28 Suppl 1:107-11. 